\title{
Single Stage Nipple-Sparing Mastectomy and Reduction Mastopexy in the Ptotic Breast
}

\author{
M. E. Pontell $\mathbb{D}^{1},{ }^{1}$ N. Saad, ${ }^{2}$ A. Brown, ${ }^{3}$ M. Rose, ${ }^{4}$ R. Ashinoff, ${ }^{4}$ and A. Saad ${ }^{4}$ \\ ${ }^{1}$ Department of Surgery, Drexel University College of Medicine, Philadelphia, PA, USA \\ ${ }^{2}$ Department of Surgery, University of Maryland, Baltimore, MD, USA \\ ${ }^{3}$ Department of Breast Surgery, Cancer Care Institute, Egg Harbor Township, NJ, USA \\ ${ }^{4}$ The Plastic Surgery Center, The Institute for Advanced Reconstruction, Egg Harbor Township, NJ, USA
}

Correspondence should be addressed to M. E. Pontell; matthewpontellmd@gmail.com

Received 25 July 2017; Revised 18 January 2018; Accepted 8 February 2018; Published 12 March 2018

Academic Editor: Nicolò Scuderi

Copyright (C) 2018 M. E. Pontell et al. This is an open access article distributed under the Creative Commons Attribution License, which permits unrestricted use, distribution, and reproduction in any medium, provided the original work is properly cited.

Purpose. Given the proposed increased risk of nipple-areolar complex (NAC) necrosis, nipple-sparing mastectomy (NSM) is generally not recommended for patients with large or significantly ptotic breasts. NAC preserving strategies in this subgroup include staged or simultaneous NSM and reduction mastopexy. We present a novel approach towards simultaneous NSM and reduction mastopexy in patients with large, ptotic breasts. Methods. Literature pertaining to NSM for women with large, ptotic breasts was reviewed and a surgical approach was designed to allow for simultaneous NSM and reduction mastopexy in such patients. Results. Eight patients underwent bilateral NSM with simultaneous reduction mammaplasty and immediate reconstruction. The majority of breasts demonstrated advanced ptosis (69\% grade III, 31\% grade II) and the average breast volume excised was 760 grams. In those patients without a history of smoking, NAC necrosis rates were $0 \%$. In those patients with a history of smoking, $83 \%$ of breasts experienced NAC necrosis (60\% total, $40 \%$ partial). One hundred percent of patients who smoked experienced some degree of NAC necrosis. Among breasts with grade II versus grade III ptosis, NAC necrosis rates were roughly equal. Conclusions. Historically, patients with large, ptotic breasts were excluded from NSM due to the proposed increased risk of NAC necrosis. This study demonstrates a safe approach towards NSM and reduction mastopexy using an inferior, wide-based, epithelialized pedicle. While all patients eventually achieved satisfactory results, there was an association between smoking and NAC necrosis. Smoking cessation is paramount to the operation's success.

\section{Introduction}

Nipple-sparing mastectomy (NSM) is a contemporary derivative of subcutaneous mastectomy (SCM), which was originally performed for fibrocystic disease of the breast $[1,2]$. NSM is an increasingly popular alternative to skinsparing mastectomy (SSM), as it allows for preservation of the nipple-areolar complex (NAC) $[1,3,4]$. With proper patient selection, NSM can be used in both the prophylactic and the therapeutic settings [1, 4-6]. Regardless of the indication, the central tenets of NSM are to remove the glandular breast tissue while maximizing structural preservation of the breast and adhering to oncologic standards $[1,3]$. The trend towards the development of more advanced NSM modifications is driven by patient demand and an increasing amount of literature documenting its therapeutic success [7].

From an oncologic perspective, NSM is reserved for patients with tumors that do not involve the skin, are less than three centimeters in diameter, and are at least two centimeters away from the NAC $[4,8]$. This procedure is a safe option for the treatment of breast carcinoma, and tumor recurrence rates are low $[4,5,8-10]$. Patients with excessively large and/or ptotic breasts or clinically palpable locoregional lymphadenopathy are generally excluded from therapeutic NSM [4, 8]. From a prophylactic standpoint, bilateral mastectomy remains a point of controversy and some surgeons do advocate for its use in high-risk patients who have a strong genetic predisposition towards developing 


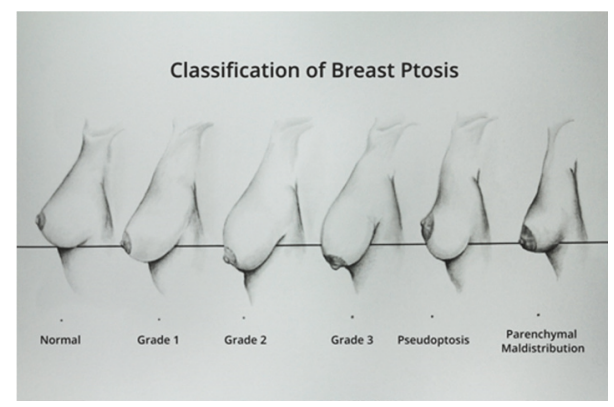

FIgURE 1: Artist's depiction of the breast ptosis grading system proposed by Regnault et al. Normal: areola above the inframammary fold (IMF) and above the gland contour; Grade I: areola at the IMF and above the gland contour; Grade II: areola below the IMF and above the gland contour; Grade III: areola below the IMF and below the gland contour; Pseudoptosis: areola at the IMF with glandular ptosis; Parenchymal Maldistribution: areola at the IMF with loose, hypoplastic glandular skin.

breast cancer $[10,11]$. On the other hand, contralateral prophylactic mastectomy (CPM) for risk reduction in patients with primary breast cancer is well supported [10, 11]. For appropriately selected patients, prophylactic mastectomy can reduce the risk of developing breast cancer by $80-95 \%$, even in the presence of a retained NAC $[4,5,10,12]$. As such, NSM is an important option in the prevention and treatment of breast cancer [12]. Additionally, NAC preservation has a positive impact on patient satisfaction $[13,14]$.

Nevertheless, the ability to perform a NSM can be restricted by patient anatomic factors. The procedure is generally not recommended for patients with breast volume exceeding 500 grams or grade II or III ptosis given the proposed increased risk of NAC necrosis (Figure 1) [4, 8]. Potential strategies for this patient subgroup include staged NSM and reduction mastopexy or NSM with simultaneous reduction mastopexy $[1,10,13,15-21]$. Here, we present an alternate way to perform a simultaneous NSM and reduction mastopexy with breast reconstruction for females with large, ptotic breasts. This technique may provide a suitable option for such women who seek NAC preservation and wish to avoid multiple operations. Additionally, implant-based reconstruction may obviate a longer procedure for those who cannot tolerate a free-flap transposition.

\section{Methods}

A review of the literature was conducted on all cases of NSM and reduction mastopexy for women with large-volume, ptotic breasts. Based on the results, a modified surgical approach was created, designed to allow for simultaneous NSM and reduction mastopexy for women with high-grade ptosis and large-volume breasts. This study was conducted under the approval of the institutional review board of AtlantiCare Medical Center. All of the NSMs were performed by a single breast surgeon $(\mathrm{AB})$ and the reconstructive procedures were performed by one, or occasionally two, of the plastic surgeons (AS, RA, and MR).
All mastectomies were nipple-sparing and were performed simultaneously with a reduction mammaplasty. Eight patients were included in this study, for a total of sixteen mastectomies $(n=16)$. Inclusion criteria consisted of patients with grade II or III breast ptosis who were candidates for prophylactic (five patients, ten breasts) or therapeutic (three patients, six breasts) NSM. After NSM and simultaneous reduction mammaplasty, patients underwent immediate placement of tissue expanders or reconstruction by deep inferior epigastric perforator (DIEP) flaps. In the tissue expander group, implants were inserted during the secondstage procedure. Additional minor revisions were made as necessary.

Data collection included patient demographics, preoperative indications, and active comorbid conditions at the time of surgery. All technical data, perioperative complications, and revision procedures were recorded and patients were followed up until all wounds had healed.

2.1. Surgical Technique. Nipple-sparing mastectomy with this technique involved a supra-areolar incision with lateral and medial extensions (Figure 2). Retroareolar breast tissue was sent for frozen section to rule out carcinoma involvement of the NAC and thin mastectomy flaps were raised superiorly and inferiorly with the NAC being thus carried on a broad, inferior-based epithelialized dermal pedicle. A variable amount of skin above the supra-areolar incision was excised in a pattern akin to a boomerang, with the width of the boomerang adjusted based upon how much lift was needed to bring the NAC into a more normal anatomic position. After raising the skin flaps up to the level of the clavicle superiorly, the inframammary fold inferiorly, the sternal border medially, and the anterior edge of the latissimus muscle laterally, the breast tissue was sharply dissected off of the pectoralis major muscle.

At this point, if expanders were used, the pectoralis major muscle was lifted off of the chest wall sharply to allow for a submuscular pocket to cover the superior and superiormedial portions of the expander. Various acellular-dermal matrix $(\mathrm{ADM})$ products were utilized to create the inferior and inferolateral coverage over the expander. Expander size was chosen based upon base width of the native breast and other chest-wall measurements. The ADM was sutured into place along the inframammary fold, the lower border of the pectoralis muscle, and the lateral chest wall with 2-0 Vicryl sutures. The expander was placed and a drain was placed below the skin but above the expander pocket. All expanders were partially inflated with sterile saline and the SPY Intraoperative Perfusion Assessment System (distributed in North America by LifeCell Corp., Branchburg, NJ; manufactured by Novadaq Technologies Inc., Richmond, British Columbia, Canada) was used at this point to confirm NAC and mastectomy flap viability. Closure consisted of two layers of 3-0 and 4-0 monocryl followed by Dermabond.

If a DIEP flap was used, then a two-team approach was used with one team member dissecting out the recipient vessels in the chest while a second team member was raising and dissecting out the DIEP flap on the abdominal wall. 


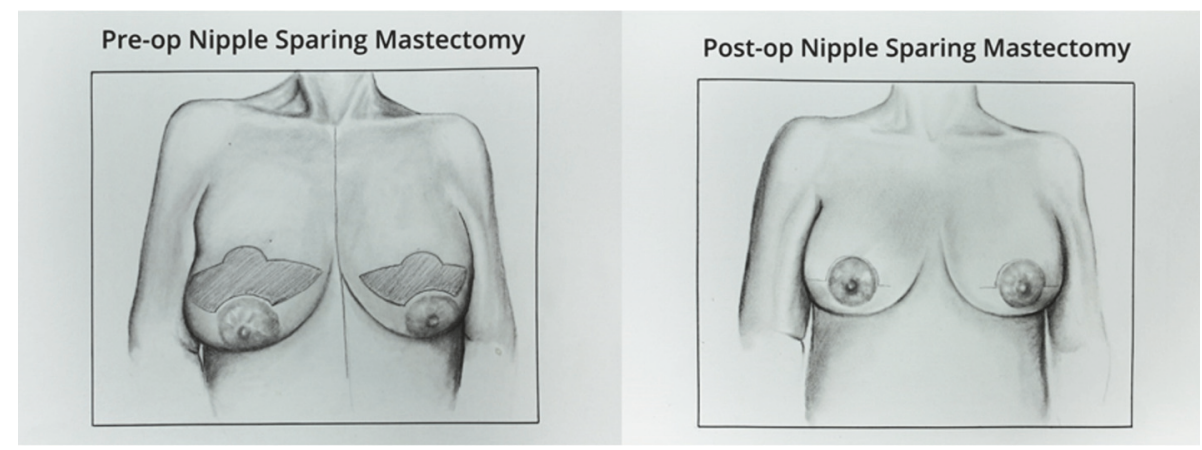

FIGURE 2: Artist's depiction of pre- and postoperative markings for simultaneous nipple-sparing mastectomy and reduction mastopexy. A "boomerang" shaped supra-areolar incision is made, through which breast tissue and a variable amount of skin are excised. The edges are reapproximated after insertion of a tissue expander.

Coupled venous anastomoses were used in all cases and handsewn arterial anastomoses were used in all cases with 8-0 nylon sutures. Flaps were stabilized onto the chest wall with 3-0 Vicryl sutures after restoration of blood flow. Abdominal fascia was repaired with 1-0 PDS sutures and the abdominal flap was closed with 0-0 PDS for the fascial layer, 3-0 monocryl for the dermal layer, and 4-0 monocryl for the skin. Ten-millimeter flat channel drains were used in the abdomen and behind the DIEP flaps in all cases. Flaps were monitored with Doppler ultrasound and clinical exam every fifteen minutes for 3 hours and then hourly thereafter.

\section{Results}

Eight patients underwent bilateral NSM with simultaneous reduction mammaplasty and breast reconstruction. A total of sixteen mastectomies were performed. Average age was 49 years, $75 \%$ of patients had comorbid conditions, and $63 \%$ of patients were actively smoking at the time of surgery. Five patients met criteria for prophylactic resection and three patients met criteria for therapeutic resection. Sixty-nine percent of breasts demonstrated grade III ptosis and the remainder were grade II. Seventy-five percent of patients had bilateral nipple-sparing mastectomies with immediate reconstruction with a tissue expander and implant insertion on a later date. The remaining $25 \%$ of patients underwent immediate reconstruction with DIEP flap. Average volume of breast tissue excised was 760 grams. In the tissue expander group, the average expander size was $560 \mathrm{cc}$ with average initial expander volumes of $240 \mathrm{cc}$ (Table 1). SPY intraoperative perfusion confirmed viable mastectomy flaps and nippleareolar complexes.

There were a total of 11 mastectomies that were not complicated by NAC necrosis. One patient developed unilateral hematoma. The average age in this group was 49 years, one patient was actively smoking at the time of surgery, and $91 \%$ of patients had active comorbid diseases. Twenty-seven percent of procedures were therapeutic, and $73 \%$ were prophylactic. Breast ptosis grades were $81 \%$ grade III and 19\% grade II. Eighty-one percent of mastectomies were reconstructed initially with tissue expanders and 19\% underwent immediate DIEP flap reconstruction. In those patients who were nonsmokers, NAC necrosis rates were $0 \%$ (Table 2).

There were a total of five mastectomies that were complicated by NAC necrosis ( $60 \%$ total, $40 \%$ partial). Of these, two breasts also developed seromas and one developed mastectomy flap necrosis. Average age in the NAC necrosis group was 59 years. All patients who developed NAC necrosis were smokers and only one patient had active comorbidities. Forty percent of procedures were prophylactic and $60 \%$ were therapeutic. Ptosis grades were $40 \%$ grade III and $60 \%$ grade II. Sixty percent of patients in this group underwent reconstruction by a tissue expander and the remaining $40 \%$ underwent immediate DIEP flap-based reconstruction (Table 3).

Rates of partial and total NAC necrosis rates were $12.5 \%$ and $18.7 \%$, respectively. Comparison of the breasts that experienced NAC necrosis with those that did not revealed average ages of 59 and 49 years, respectively. One hundred percent of patients who experienced NAC necrosis were smokers versus $9 \%$ in the NAC intact group. Twenty percent of cases of NAC necrosis had associated comorbidities versus $91 \%$ in the NAC intact group. On average, the percentage of therapeutic mastectomies was slightly higher in the NAC necrosis group; however, the percentage of grade III ptosis was lower. Reconstruction methods were similar in both groups (Table 4). All patients were eventually able to heal their incisions and postoperative wounds (Figure 3 ).

\section{Discussion}

Female patients with large-volume, severely ptotic breasts who are candidates for NSM pose a specific challenge to reconstructive surgeons. Most surgeons are reluctant to perform a simultaneous NSM and reduction mastopexy given the supposed increased risk of NAC and skin flap necrosis $[1,4,7,12,13,22]$. Some authors argue that advanced breast ptosis may further contribute to the development of this complication and may also impair NAC repositioning and management of the skin envelope when necessary $[1,4$, $7,12,22]$. Studies propose that high-grade ptosis and/or 
TABLE 1: Patient characteristics and procedural specifics.

\begin{tabular}{|c|c|c|c|c|c|c|c|c|c|}
\hline $\mathrm{Pt}$ & Age & Sex & Smoker & $\mathrm{PMH}$ & Indication & Ptosis grade & Technique & $\begin{array}{l}\text { Expander } \\
\text { size }\end{array}$ & $\begin{array}{l}\text { Breast volume } \\
\text { excised }(\mathrm{R} / \mathrm{L})\end{array}$ \\
\hline 1 & 55 & $\mathrm{~F}$ & No & Hypertension & $\begin{array}{l}\text { Biopsy with atypical } \\
\text { cells in the setting of } \\
\text { bilateral silicone } \\
\text { injections }\end{array}$ & $\begin{array}{c}\text { III } \\
(B / L)\end{array}$ & $\begin{array}{l}\text { Bilateral NSM with } \\
\text { reduction mammaplasty } \\
\text { and expander insertion }\end{array}$ & $350 \mathrm{cc}$ & $665 \mathrm{gr} / 740 \mathrm{gr}$ \\
\hline 2 & 30 & $\mathrm{~F}$ & No & $\begin{array}{l}\text { Asthma, } \\
\text { depression }\end{array}$ & BRCA mutation & $\begin{array}{c}\mathrm{III} \\
(\mathrm{B} / \mathrm{L})\end{array}$ & $\begin{array}{l}\text { Bilateral NSM with } \\
\text { reduction mammaplasty } \\
\text { and expander insertion }\end{array}$ & $800 \mathrm{cc}$ & $1240 \mathrm{gr} / 1316 \mathrm{gr}$ \\
\hline 3 & 54 & $\mathrm{~F}$ & No & $\begin{array}{l}\text { Gastric cancer, } \\
\text { thyroid disease, } \\
\text { peripheral } \\
\text { neuropathy }\end{array}$ & BRCA mutation & $\begin{array}{c}\text { III } \\
(B / L)\end{array}$ & $\begin{array}{l}\text { Bilateral NSM with } \\
\text { reduction mammaplasty } \\
\text { and expander insertion }\end{array}$ & $400 \mathrm{cc}$ & $429 \mathrm{gr} / 449 \mathrm{gr}$ \\
\hline 4 & 58 & $\mathrm{~F}$ & No & Thyroid disease & BRCA mutation & $\begin{array}{c}\text { III } \\
(\mathrm{B} / \mathrm{L})\end{array}$ & $\begin{array}{l}\text { Bilateral NSM with } \\
\text { reduction mammaplasty } \\
\text { and expander insertion }\end{array}$ & $800 \mathrm{cc}$ & $1006 \mathrm{gr} / 776 \mathrm{gr}$ \\
\hline 5 & 52 & $\mathrm{~F}$ & Yes & None & $\begin{array}{l}\text { Unilateral, multifocal } \\
\text { DCIS }\end{array}$ & $\begin{array}{c}\text { III } \\
(\mathrm{B} / \mathrm{L})\end{array}$ & $\begin{array}{l}\text { Bilateral NSM with } \\
\text { reduction mammaplasty } \\
\text { and DIEP flap } \\
\text { reconstruction }\end{array}$ & N/A & NR \\
\hline 6 & 58 & $\mathrm{~F}$ & No & $\begin{array}{l}\text { Hypertension, } \\
\text { diabetes mellitus }\end{array}$ & $\begin{array}{l}\text { Unilateral invasive } \\
\text { breast cancer, BRCA }\end{array}$ & $\begin{array}{l}\mathrm{III} / \mathrm{II} \\
(\mathrm{R} / \mathrm{L})\end{array}$ & $\begin{array}{l}\text { Bilateral NSM with } \\
\text { reduction mammaplasty } \\
\text { and DIEP flap } \\
\text { reconstruction }\end{array}$ & N/A & $546 \mathrm{gr} / 436 \mathrm{gr}$ \\
\hline 7 & 32 & $\mathrm{~F}$ & Yes & None & $\begin{array}{l}\text { Unilateral invasive } \\
\text { breast cancer }\end{array}$ & $\begin{array}{c}\mathrm{II} \\
(\mathrm{B} / \mathrm{L})\end{array}$ & $\begin{array}{l}\text { Bilateral NSM with } \\
\text { reduction mammaplasty } \\
\text { and expander insertion }\end{array}$ & $500 \mathrm{cc}$ & NR \\
\hline 8 & 55 & $\mathrm{~F}$ & Yes & $\begin{array}{l}\text { Ovarian cancer, } \\
\text { thyroid disease }\end{array}$ & BRCA & $\begin{array}{c}\mathrm{II} \\
(\mathrm{B} / \mathrm{L})\end{array}$ & $\begin{array}{l}\text { Bilateral NSM with } \\
\text { reduction mammaplasty } \\
\text { and expander insertion }\end{array}$ & $500 \mathrm{cc}$ & NR \\
\hline
\end{tabular}

PMH: past medical history; R: right; L: left; B/L: bilateral; NSM: nipple-sparing mastectomy; BRCA: breast cancer susceptibility gene; DCIS: ductal carcinoma in situ; N/A: not applicable; DIEP: deep inferior epigastric perforator; NR: not reported.

TABLE 2: Breasts that did not experience NAC necrosis stratified by individual mastectomy.

\begin{tabular}{|c|c|c|c|c|c|c|c|c|}
\hline Pt. & NAC necrosis & $\begin{array}{c}\text { Wound } \\
\text { complications }\end{array}$ & Age & Smoker & $\mathrm{PMH}$ & Indication & Ptosis grade & Reconstruction \\
\hline $1(\mathrm{R})$ & No & Hematoma & 55 & $\mathrm{~N}$ & $\mathrm{Y}$ & Prophylactic & III & Expander \\
\hline $1(\mathrm{~L})$ & No & None & 55 & $\mathrm{~N}$ & $\mathrm{Y}$ & Prophylactic & III & Expander \\
\hline $2(\mathrm{R})$ & No & None & 30 & $\mathrm{~N}$ & $\mathrm{Y}$ & Prophylactic & III & Expander \\
\hline $2(\mathrm{~L})$ & No & None & 30 & $\mathrm{~N}$ & $\mathrm{Y}$ & Prophylactic & III & Expander \\
\hline $3(\mathrm{R})$ & No & None & 54 & $\mathrm{~N}$ & $\mathrm{Y}$ & Prophylactic & III & Expander \\
\hline $3(\mathrm{~L})$ & No & None & 54 & $\mathrm{~N}$ & $\mathrm{Y}$ & Prophylactic & III & Expander \\
\hline $4(\mathrm{R})$ & No & None & 58 & $\mathrm{~N}$ & $\mathrm{Y}$ & Prophylactic & III & Expander \\
\hline $4(\mathrm{~L})$ & No & None & 58 & $\mathrm{~N}$ & $\mathrm{Y}$ & Prophylactic & III & Expander \\
\hline $6(\mathrm{~L})$ & No & None & 58 & $\mathrm{~N}$ & $\mathrm{Y}$ & Therapeutic & II & DIEP \\
\hline $6(\mathrm{R})$ & No & None & 58 & $\mathrm{~N}$ & $\mathrm{Y}$ & Therapeutic & III & DIEP \\
\hline $7(\mathrm{~L})$ & No & None & 32 & $\mathrm{Y}$ & $\mathrm{N}$ & Therapeutic & II & Expander \\
\hline
\end{tabular}

Pt.: patient number; NAC: nipple-areolar complex; PMH: past medical history; N: no; Y: yes; R: right breast; L: left breast; DIEP: deep inferior epigastric perforator.

excessive breast volume may increase the length of the skin flap required to supply the NAC, thereby compromising vascular supply [22]. Additionally, some argue that substantial amounts of breast tissue need to be left behind to ensure NAC and flap perfusion resulting in an inadequate mastectomy [13]. Nevertheless, several studies have reported options for women with large breasts and/or advanced ptosis who meet criteria for NSM $[1,13]$. These techniques can be broadly 
TABLE 3: Breasts that experienced NAC necrosis stratified by individual mastectomy.

\begin{tabular}{lccccccrr}
\hline Pt. & NAC necrosis & $\begin{array}{c}\text { Wound } \\
\text { complications }\end{array}$ & Age & Smoker & PMH & Indication & Ptosis grade & Reconstruction \\
\hline $5(\mathrm{R})$ & Partial & None & 52 & $\mathrm{Y}$ & $\mathrm{N}$ & Therapeutic & III & DIEP \\
$5(\mathrm{~L})$ & Total & Flap necrosis & 52 & $\mathrm{Y}$ & $\mathrm{N}$ & Therapeutic & III & DIEP \\
$7(\mathrm{R})$ & Partial & None & 32 & $\mathrm{Y}$ & $\mathrm{N}$ & Therapeutic & II & Expander \\
$8(\mathrm{R})$ & Total & Seroma & 55 & $\mathrm{Y}$ & $\mathrm{Y}$ & Prophylactic & II & Expander \\
$8(\mathrm{~L})$ & Total & Seroma & 55 & $\mathrm{Y}$ & $\mathrm{Y}$ & Prophylactic & II & Expander \\
\hline
\end{tabular}

Pt.: patient number; NAC: nipple-areolar complex; R: right breast; L: left breast; Y: yes; N: no; DIEP: deep inferior epigastric perforator.

TABLE 4: Breasts that experienced NAC necrosis compared to those that did not.

\begin{tabular}{lccccccc}
\hline Group & $\begin{array}{c}\text { Number of } \\
\text { breasts }\end{array}$ & Avg. age & Smokers & $\begin{array}{c}\text { Comorbidities } \\
\text { present }\end{array}$ & $\begin{array}{c}\text { Therapeutic versus } \\
\text { prophylactic }\end{array}$ & $\begin{array}{c}\text { Ptosis grade } \\
\text { (II versus III) }\end{array}$ & $\begin{array}{c}\text { Expander } \\
\text { versus DIEP }\end{array}$ \\
\hline NAC necrosis & 5 & 59 years & $100 \%$ & $20 \%$ & $60 \%$ versus $40 \%$ & $\begin{array}{c}60 \% \text { versus } \\
40 \%\end{array}$ & $\begin{array}{c}60 \% \text { versus } \\
40 \%\end{array}$ \\
NAC intact & 11 & 49 years & $9 \%$ & $91 \%$ & $27 \%$ versus $73 \%$ & $\begin{array}{c}19 \% \text { versus } \\
81 \%\end{array}$ & $\begin{array}{c}81 \% \text { versus } \\
19 \%\end{array}$ \\
\hline
\end{tabular}

NAC: nipple-areolar complex; Avg.: average; DIEP: deep inferior epigastric perforator.

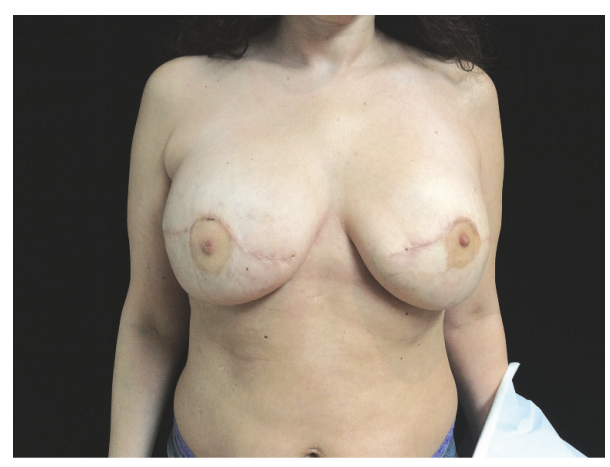

(a)

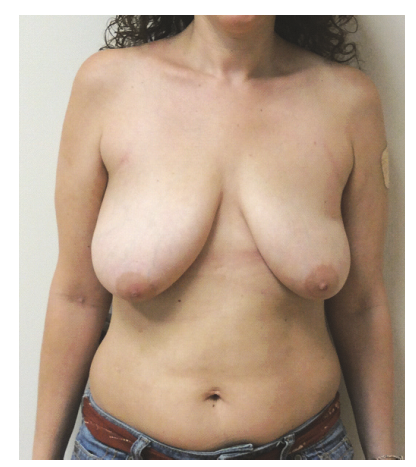

(b)

FIGURE 3: Pre- (a) and postoperative (b) photographs after simultaneous nipple-sparing mastectomy and reduction mastopexy with implantbased reconstruction.

subdivided into staged NSM and reduction mastopexy $[1,13$, $23]$ or simultaneous reduction mastopexy with NSM [10, 1621].

Review of the literature revealed three studies that focused on staged reduction mastopexy and NSM for women with large, ptotic breasts $[1,13,23]$. Spear et al. published a series of cases in which such patients were offered staged NSM and reduction mastopexy [13]. Partial NAC necrosis rates were roughly $12.5 \%$ and there were no cases of total NAC necrosis [13]. While results were promising, the authors felt this procedure would be best suited for patients with medium-volume breasts with moderate ptosis [13]. Two other studies employed the use of immediate flap-based reconstruction after NSM with a delayed reduction mastopexy $[1,23]$. These studies used various free flaps to support NAC perfusion after NSM, and reduction mastopexy for moderately to severely ptotic breasts was performed on a later date $[1,23]$. The main disadvantage in the staged approach is that the patient requires two major surgeries. Additionally, patients with active comorbid conditions may not be able to tolerate a lengthy free-flap procedure. The mean percentages of partial and total NAC necrosis in the staged group were $4.16 \%$ and $1 \%$, respectively (Table 5 ).

In the 1970s, several studies examined the utility of SCM with NAC preservation with simultaneous reduction mastopexy for patients with large breasts and/or severe ptosis $[16-19,21]$. While these studies showed promising results regarding NAC preservation, several failed to specify the breast size or degree of ptosis [16, 19]. Additionally, early studies focused on SCM with NAC preservation, which likely resulted in a less comprehensive mastectomy as indications at that time were strictly prophylactic. The literature suggests that breast tissue quantities now considered unacceptable for conventional NSM were left behind during SCM to support NAC and flap perfusion [1]. Nevertheless, there has been a resurgence of interest in simultaneous NSM and reduction mastopexy, likely reflecting the increase in patient demand [7]. Two studies in 2010 and 2011 demonstrated good results 
TABLE 5: Table reviewing all of the studies published on nipple-sparing mastectomy in large-volume, ptotic breasts from 1970 to 2016.

\begin{tabular}{|c|c|c|c|c|c|c|c|c|}
\hline & Technique & Reconstruction & $\begin{array}{l}\text { Sample } \\
\text { size } \\
\text { (num- } \\
\text { ber of } \\
\text { breasts) }\end{array}$ & Indication & $\begin{array}{l}\text { Ptosis/breast } \\
\text { volume }\end{array}$ & $\begin{array}{l}\text { Partial } \\
\text { NAC } \\
\text { necrosis }\end{array}$ & $\begin{array}{c}\text { Total } \\
\text { NAC } \\
\text { necrosis }\end{array}$ & Other complications \\
\hline \multicolumn{9}{|c|}{ Simultaneous mastopexy and NSM } \\
\hline $\begin{array}{l}\text { Goulian \& } \\
\text { McDivitt, } 1972\end{array}$ & $\begin{array}{l}\text { SCM with } \\
\text { reduction } \\
\text { mastopexy }\end{array}$ & $\begin{array}{l}\text { Implant } \\
\text { None }\end{array}$ & 24 & $\begin{array}{l}\text { Risk } \\
\text { reduction }\end{array}$ & $\begin{array}{c}\text { Medium- } \\
\text { large } \\
\text { Not specified }\end{array}$ & None & None & Hematoma (NR) \\
\hline Biggs et al., 1977 & $\begin{array}{l}\text { SCM with } \\
\text { reduction } \\
\text { mastopexy }\end{array}$ & Implant & 33 & NR & $\begin{array}{l}\text { Not specified } \\
\text { Min-Mod } \\
\text { ptosis }\end{array}$ & None & None & $\begin{array}{c}\text { Partial flap necrosis } \\
\text { (1) } \\
\text { Capsular contractures } \\
(8) \\
\text { Atrophy requiring } \\
\text { excision (1) } \\
\text { Explant (1) }\end{array}$ \\
\hline Jarrett et al., 1978 & $\begin{array}{l}\text { SCM, reduction } \\
\text { mastopexy, and } \\
\text { free nipple graft }\end{array}$ & Implant & 44 & $\begin{array}{l}\text { Risk } \\
\text { reduction }\end{array}$ & $\begin{array}{l}\text { Large volume } \\
\text { Severe ptosis }\end{array}$ & None & None & NR \\
\hline Gibson, 1979 & $\begin{array}{l}\text { SCM with } \\
\text { reduction } \\
\text { mastopexy }\end{array}$ & NR & NR & $\begin{array}{l}\text { Risk } \\
\text { reduction }\end{array}$ & Not specified & None & None & NR \\
\hline Rusby \& Gui, 2010 & $\begin{array}{l}\text { NSM with } \\
\text { reduction } \\
\text { mastopexy }\end{array}$ & Expander & 16 & $\begin{array}{l}\text { Risk } \\
\text { reduction }\end{array}$ & $\begin{array}{l}\text { NR } \\
\text { NR }\end{array}$ & NR & $6.3 \%$ & None \\
\hline Nava et al., 2011 & $\begin{array}{l}\text { NSM with } \\
\text { reduction } \\
\text { mastopexy }\end{array}$ & Implant & 13 & Therapeutic & $\begin{array}{l}\text { NR } \\
\text { NR }\end{array}$ & $\mathrm{NR}^{\#}$ & $\mathrm{NR}^{\#}$ & $\mathrm{NR}^{\#}$ \\
\hline Rivolin et al., 2012 & $\begin{array}{c}\text { NSM with } \\
\text { periareolar pexy }\end{array}$ & Implant & 22 & Therapeutic & $\begin{array}{l}\text { Medium- } \\
\text { large volume } \\
\text { Moderate } \\
\text { ptosis }\end{array}$ & $13.6 \%$ & $4.6 \%{ }^{*}$ & None \\
\hline $\begin{array}{l}\text { Al-Mufarrej et al., } \\
2013\end{array}$ & $\begin{array}{l}\text { NSM with } \\
\text { reduction } \\
\text { mastopexy }\end{array}$ & $\begin{array}{l}\text { Expander } \\
\text { Implant }\end{array}$ & 48 & $\begin{array}{l}\text { Risk } \\
\text { reduction }\end{array}$ & $\begin{array}{l}\text { Large volume } \\
\text { Moderate }\end{array}$ & $8.3 \%$ & $4.2 \%$ & $\begin{array}{c}\text { Infected implant } \\
(2.1 \%) \\
\text { Implant rupture } \\
(14.6 \%) \\
\text { Hematoma }(2.1 \%) \\
\text { Capsular contracture } \\
(4.2 \%) \\
\end{array}$ \\
\hline $\begin{array}{l}\text { Pontell et al., } 2016 \\
\text { (this report) }\end{array}$ & $\begin{array}{l}\text { NSM with } \\
\text { reduction } \\
\text { mastopexy }\end{array}$ & $\begin{array}{l}\text { Expander } \\
\text { DIEP Flap }\end{array}$ & 16 & $\begin{array}{c}\text { Risk } \\
\text { reduction } \\
\text { Therapeutic }\end{array}$ & $\begin{array}{l}\text { Large volume } \\
\text { Grade II/III } \\
\text { ptosis }\end{array}$ & $0 \%{ }^{* *}$ & $0 \%{ }^{* *}$ & $\begin{array}{c}\text { Hematoma } \\
\text { Seroma } \\
\text { Mastectomy flap } \\
\text { necrosis }\end{array}$ \\
\hline \multicolumn{9}{|c|}{ Staged mastopexy and NSM } \\
\hline $\begin{array}{l}\text { Schneider et al., } \\
2012\end{array}$ & $\begin{array}{c}\text { NSM with } \\
\text { immediate flap } \\
\text { placement and } \\
\text { staged reduction } \\
\text { mastopexy }\end{array}$ & $\begin{array}{l}\text { TUG Flap } \\
\text { DIEP Flap }\end{array}$ & 34 & NR & $\begin{array}{l}\text { Large volume } \\
\text { Grade II/III } \\
\text { ptosis }\end{array}$ & None & $3 \%$ & Hematoma (3\%) \\
\hline $\begin{array}{l}\text { DellaCroce et al., } \\
2015\end{array}$ & $\begin{array}{l}\text { NSM with } \\
\text { immediate flap } \\
\text { placement and } \\
\text { staged reduction } \\
\text { mastopexy }\end{array}$ & $\begin{array}{l}\text { DIEP Flap } \\
\text { SGAP Flap }\end{array}$ & 110 & $\begin{array}{c}\text { Risk } \\
\text { reduction } \\
\text { Therapeutic }\end{array}$ & $\begin{array}{l}\text { Medium- } \\
\text { large volume } \\
\text { Grade II/III } \\
\text { ptosis }\end{array}$ & None & None & $\begin{array}{c}\text { Partial mastectomy } \\
\text { flap necrosis }(3.6 \%) \\
\text { Incisional dehiscence } \\
(8 \%) \\
\text { Hematoma }(2.7 \%) \\
\text { Partial flap necrosis } \\
(1.8 \%)\end{array}$ \\
\hline
\end{tabular}


TABLE 5: Continued.

\begin{tabular}{|c|c|c|c|c|c|c|c|c|}
\hline & Technique & Reconstruction & $\begin{array}{c}\text { Sample } \\
\text { size } \\
\text { (num- } \\
\text { ber of } \\
\text { breasts) }\end{array}$ & Indication & $\begin{array}{c}\text { Ptosis/breast } \\
\text { volume }\end{array}$ & $\begin{array}{l}\text { Partial } \\
\text { NAC } \\
\text { necrosis }\end{array}$ & $\begin{array}{c}\text { Total } \\
\text { NAC } \\
\text { necrosis }\end{array}$ & Other complications \\
\hline Spear et al., 2012 & $\begin{array}{c}\text { Reduction } \\
\text { mastopexy } \\
\text { followed by } \\
\text { NSM }\end{array}$ & $\begin{array}{c}\text { Implant } \\
\text { Tissue expander }\end{array}$ & 24 & $\begin{array}{c}\text { Risk } \\
\text { reduction } \\
\text { Therapeutic }\end{array}$ & $\begin{array}{l}\text { Medium } \\
\text { volume } \\
\text { Grade II/III } \\
\text { ptosis }\end{array}$ & $12.5 \%$ & None & $\begin{array}{c}\text { Breast infection }(8 \%) \\
\text { Skin flap necrosis } \\
(17 \%) \\
\text { Explant }(4 \%)\end{array}$ \\
\hline
\end{tabular}

NAC: nipple-areolar complex; NSM: nipple-sparing mastectomy; SCM: subcutaneous mastectomy; NR: not reported; DIEP: deep inferior epigastric perforator; TUG: transverse upper gracilis; SGAP: superior gluteal artery perforator. ${ }^{*}$ Complications were not stratified by NSM (SRM) versus SSM status. ${ }^{*}$ This study mentions the exclusion of one patient who had total NAC necrosis. ${ }^{* *}$ These rates exclude the patients who were smokers, including patients with partial and total NAC necrosis rates of $12.5 \%$ and $18.7 \%$, respectively.

regarding NAC preservation; however, the data reported did not allow any conclusions to be drawn regarding the breast size or degree of ptosis [7, 24]. Al-Mufarrej et al. and Rivolin et al. reported on two series of patients with medium- to large-volume breasts and moderate ptosis who underwent NSM with simultaneous reduction mastopexy $[10,15]$. Results of these studies demonstrated excellent NAC preservation in prophylactic and therapeutic scenarios; however, the issue of severe breast ptosis did not appear to be addressed $[10,15]$. Average partial and total NAC necrosis rates in the simultaneous group were $3.65 \%$ and $2.16 \%$, respectively (Table 5).

The pedicled flap used in this study is a wide-based, epithelialized version of the traditional inferiorly based flaps used during reduction mammaplasty. The base of the flap was widened in attempt to preserve the natural arterial and venous supply to the NAC. The NAC receives arterial perfusion from a periareolar network that is supplied by perforating branches of the internal thoracic artery, the anterior intercostal arteries, and the lateral thoracic artery [25]. The most important contribution arises from the third internal thoracic artery perforator [25]. All of these arterial networks course towards the NAC in a medio- or lateroinferior direction (Figure 4). After formation of the periareolar plexus, the cutaneous perforators travel within the subcutaneous tissue before reaching the NAC and after mastectomy the NAC relies solely on these cutaneous branches as the underlying breast tissue has been removed $[1,26]$. With respect to vascular outflow, the NAC is drained through a superior and inferior horizontal venous sling (Figure 4) [27]. After mastectomy, the NAC drainage relies heavily on the superficial, inferiorly coursing venous network [27]. The cutaneous venous system is even more superficial than the arterial network and as such is more likely to be damaged during deepithelialization [27]. Necrosis of the NAC results from either arterial or venous insufficiency and the latter appears to be even more prevalent with larger breast resection volumes [13, 27-29]. Given the vascular anatomy of the NAC, expanding the base of the pedicle in a lateral fashion should theoretically preserve more of the arterial supply and venous drainage. In addition, by maintaining an epithelialized pedicle, the cutaneous vascular perforators that nourish the NAC should also be better preserved. The importance of

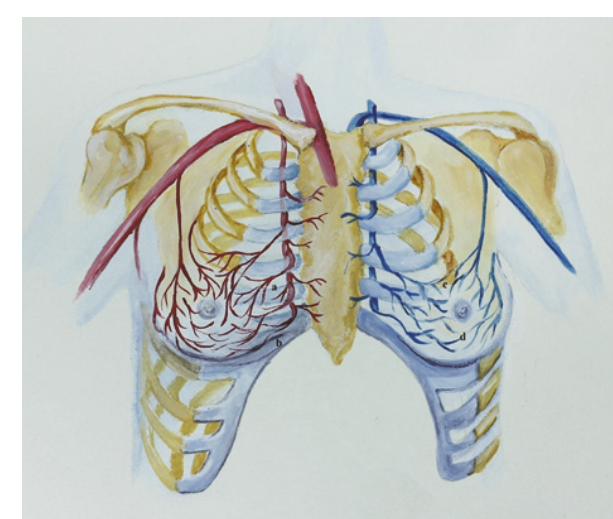

Figure 4: Artist's depiction of the arterial supply (right breast) and venous drainage (left breast) to the nipple-areolar complex (NAC). The most important contributor to NAC perfusion arises from the third internal thoracic artery perforator (a). This branch travels medially from its origin and courses just under the NAC where it gives off tributaries to the periareolar network. The anterior intercostal arteries originate more inferiorly and course along the inframammary fold before giving their contributions to the arterial supply of the NAC (b). The NAC is drained through superior (c) and inferior (d) horizontal venous slings that ultimately drain into the thoracic and subclavian veins $[17,19]$.

vascular preservation is amplified with larger breast volumes $[27,28]$. In theory, such dissection should offer anatomical advantages when compared to other techniques that use narrow, deepithelialized pedicles to support NAC perfusion after NSM and reduction mammaplasty.

Limitations of this study include the small sample size and thus an inability to draw statistically significant conclusions. In addition, while the average breast volume excised was 760 grams, several of the patients did not have excised breast volumes recorded and therefore NAC necrosis could not be analyzed alongside breast volumes. Overall rates of partial and total NAC necrosis were $12.5 \%$ and $18.7 \%$, respectively. The discordance between SPY perfusion results and NAC survival may represent either a lack of diagnostic accuracy on behalf of the SPY system or, more likely, the complex microvascular disease that develops in active smokers. While these complication rates do appear high, subset analysis 
reveals that all patients who had NAC necrosis were smokers and all patients who smoked developed NAC necrosis. Excluding the subset of patients who smoked, partial and total NAC necrosis rates were $0 \%$. Such complications did not appear to be related to patient age, the presence or absence of comorbidities, indication for procedure, grade II versus III ptosis, or the type of reconstruction performed.

In summary, this study presents an alternate technique for simultaneous NSM and reduction mastopexy for women with large, ptotic breasts. Using this method, comparable amounts of NAC preservation were able to be achieved in what has historically been considered a high-risk patient group for this procedure. While NSM has traditionally been avoided in this patient subgroup, this study supports its inclusion when considering a patient for either prophylactic or therapeutic NSM. Using a wide, inferior, epithelialized pedicle based on the vascular anatomy of the NAC, comparable rates of NAC preservation are possible, even in patients with large-volume, severely ptotic breasts. Options for immediate reconstruction exist, and a staged approach may not be necessary. Emphasis on smoking cessation is paramount to the success of the operation.

\section{Conflicts of Interest}

The authors declare that they have no conflicts of interest.

\section{References}

[1] F. J. DellaCroce, C. A. Blum, S. K. Sullivan et al., "Nipple-Sparing Mastectomy and Ptosis: Perforator Flap Breast Reconstruction Allows Full Secondary Mastopexy with Complete Nipple Areolar Repositioning," Plastic and Reconstructive Surgery, vol. 136, no. 1, pp. 1-9, 2015.

[2] S. Spear, "Nipple sparing mastectomy and reconstruction: Indications, techniques, and outcomes," in Surgery of the Breast: Principles and Art, S. Spear, Ed., vol. 1, pp. 287-297, Wolters Kluwer/Lippincott WIlliams \& Wilkins, 3rd edition, 2011.

[3] H. R. Moyer, B. Ghazi, J. R. Daniel, R. Gasgarth, and G. W. Carlson, "Nipple-sparing mastectomy: Technical aspects and aesthetic outcomes," Annals of Plastic Surgery, vol. 68, no. 5, pp. 446-450, 2012.

[4] S. L. Spear, S. C. Willey, E. D. Feldman et al., "Nipple-sparing mastectomy for prophylactic and therapeutic indications," Plastic and Reconstructive Surgery, vol. 128, no. 5, pp. 1005-1014, 2011.

[5] L. C. Hartmann, D. J. Schaid, J. E. Woods et al., "Efficacy of bilateral prophylactic mastectomy in women with a family history of breast cancer," The New England Journal of Medicine, vol. 340, no. 2, pp. 77-84, 1999.

[6] P. Maxwell, P. Whitworth, and A. Gabriel, "Nipple sparing mastectomy," in Surgery of the Breast: Principles and Art, S. Spear, Ed., vol. 1, pp. 298-307, Wolters Kluwer/Lippincott WIlliams \& Wilkins, 3rd edition, 2011.

[7] JE. Rusby and GP. Gui, "Nipple-sparing mastectomy in women with large or ptotic breasts," Journal of Plastic, Reconstructive and Aesthetic Surgery, vol. 125, pp. 818-829, 2010.

[8] S. L. Spear, C. M. Hannan, S. C. Willey, and C. Cocilovo, "Nipple-sparing mastectomy," Plastic and Reconstructive Surgery, vol. 123, no. 6, pp. 1665-1673, 2009.
[9] C. M. Chen, J. J. Disa, V. Sacchini et al., "Nipple-sparing mastectomy and immediate tissue expander/implant breast reconstruction," Plastic and Reconstructive Surgery, vol. 124, no. 6, pp. 1772-1780, 2009.

[10] F. M. Al-Mufarrej, J. E. Woods, and S. R. Jacobson, "Simultaneous mastopexy in patients undergoing prophylactic nipplesparing mastectomies and immediate reconstruction," Journal of Plastic, Reconstructive \& Aesthetic Surgery, vol. 66, no. 6, pp. 747-755, 2013.

[11] L. Lostumbo, N. E. Carbine, and J. Wallace, "Prophylactic mastectomy for the prevention of breast cancer," Cochrane Database of Systematic Reviews, vol. 11, Article ID CD002748, 2010.

[12] S. L. Spear, M. E. Carter, and K. Schwarz, "Prophylactic mastectomy: Indications, options, and reconstructive alternatives," Plastic and Reconstructive Surgery, vol. 115, no. 3, pp. 891-909, 2005.

[13] S. L. Spear, S. J. Rottman, L. A. Seiboth, and C. M. Hannan, "Breast reconstruction using a staged nipple-sparing mastectomy following mastopexy or reduction," Plastic and Reconstructive Surgery, vol. 129, no. 3, pp. 572-581, 2012.

[14] D. K. Wellisch, W. S. Schain, R. Barrett Noone, and J. W. Little, "The psychological contribution of nipple addition in breast reconstruction," Plastic and Reconstructive Surgery, vol. 80, no. 5, pp. 699-704, 1987.

[15] A. Rivolin, F. Kubatzki, F. Marocco et al., "Nipple-areola complex sparing mastectomy with periareolar pexy for breast cancer patients with moderately ptotic breasts," Journal of Plastic, Reconstructive \& Aesthetic Surgery, vol. 65, no. 3, pp. 296-303, 2012.

[16] E. W. GIBSON, "Subcutaneous mastectomy using an inferior nipple pedicle," ANZ Journal of Surgery, vol. 49, no. 5, pp. 559560, 1979.

[17] J. E. Woods, "Detailed technique of subcutaneous mastectomy with and without mastopexy," Annals of Plastic Surgery, vol. 18, no. 1, pp. 51-61, 1987.

[18] J. R. Jarrett, R. G. Cutler, and D. F. Teal, "Subcutaneous mastectomy in small, large, or ptotic breasts with immediate submuscular placement of implants," Plastic and Reconstructive Surgery, vol. 62, no. 5, pp. 702-705, 1978.

[19] D. Goulian and R. W. McDivitt, "Subcutaneous mastectomy with immediate reconstruction of the breasts, using the dermal mastopexy technique," Plastic and Reconstructive Surgery, vol. 50, no. 3, pp. 211-215, 1972.

[20] N. G. Georgiade and W. Hyland, "Technique for subcutaneous mastectomy and immediate reconstruction in the ptotic breast," Plastic and Reconstructive Surgery, vol. 56, no. 2, pp. 121-128, 1975.

[21] T. M. Biggs, R. O. Brauer, and L. E. Wolf, "Mastopexy in conjunction with subcutaneous mastectomy," Plastic and Reconstructive Surgery, vol. 60, no. 1, pp. 1-5, 1977.

[22] P. Chirappapha, J.-Y. Petit, M. Rietjens et al., "Nipple sparing mastectomy: Does breast morphological factor related to necrotic complications?" Plastic and Reconstructive Surgery, vol. 2, no. e99, pp. 1-7, 2014.

[23] L. F. Schneider, C. M. Chen, A. J. Stolier, R. L. Shapiro, C. Y. Ahn, and R. J. Allen, "Nipple-sparing mastectomy and immediate free-flap reconstruction in the large ptotic breast," Annals of Plastic Surgery, vol. 69, no. 4, pp. 425-428, 2012.

[24] M. B. Nava, J. Ottolenghi, A. Pennati et al., "Skin/nipple sparing mastectomies and implant-based breast reconstruction in 
patients with large and ptotic breast: oncological and reconstructive results," The Breast, vol. 21, no. 3, pp. 267-271, 2012.

[25] P. V. van Deventer, "The blood supply to the nipple-areola complex of the human mammary gland," Aesthetic Plastic Surgery, vol. 28, no. 6, pp. 393-398, 2004.

[26] H. Nakajima, N. Imanishi, and S. Aiso, "Arterial anatomy of the nipple-areola complex," Plastic and Reconstructive Surgery, vol. 96, no. 4, pp. 843-845, 1995.

[27] C. M. Le Roux, W.-R. Pan, S. A. Matousek, and M. W. Ashton, "Preventing venous congestion of the nipple-areola complex: An anatomical guide to preserving essential venous drainage networks," Plastic and Reconstructive Surgery, vol. 127, no. 3, pp. 1073-1079, 2011.

[28] G. Gravante, A. Araco, R. Sorge et al., "Postoperative wound infections after breast reductions: The role of smoking and the amount of tissue removed," Aesthetic Plastic Surgery, vol. 32, no. 1, pp. 25-31, 2008.

[29] P. V. van Deventer, B. J. Page, and F. R. Graewe, "The safety of pedicles in breast reduction and mastopexy procedures," Aesthetic Plastic Surgery, vol. 32, no. 2, pp. 307-312, 2008. 


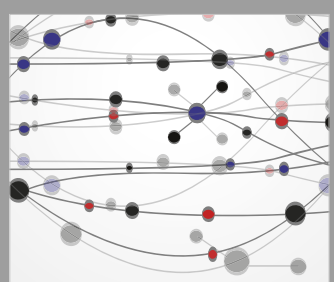

The Scientific World Journal
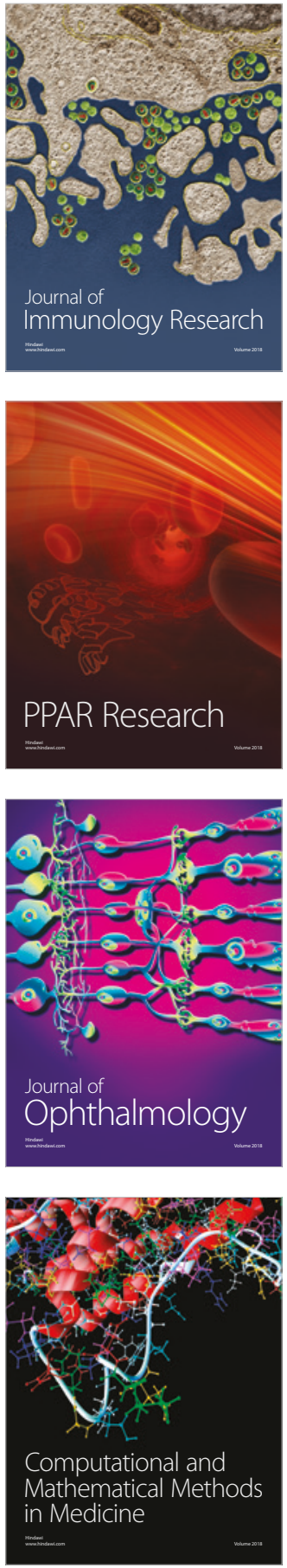

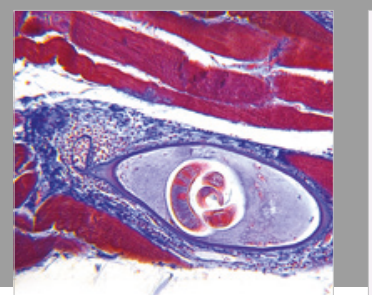

Gastroenterology Research and Practice

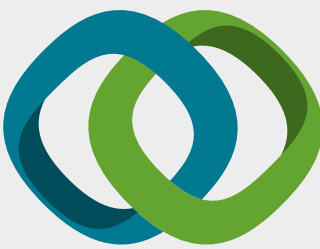

\section{Hindawi}

Submit your manuscripts at

www.hindawi.com
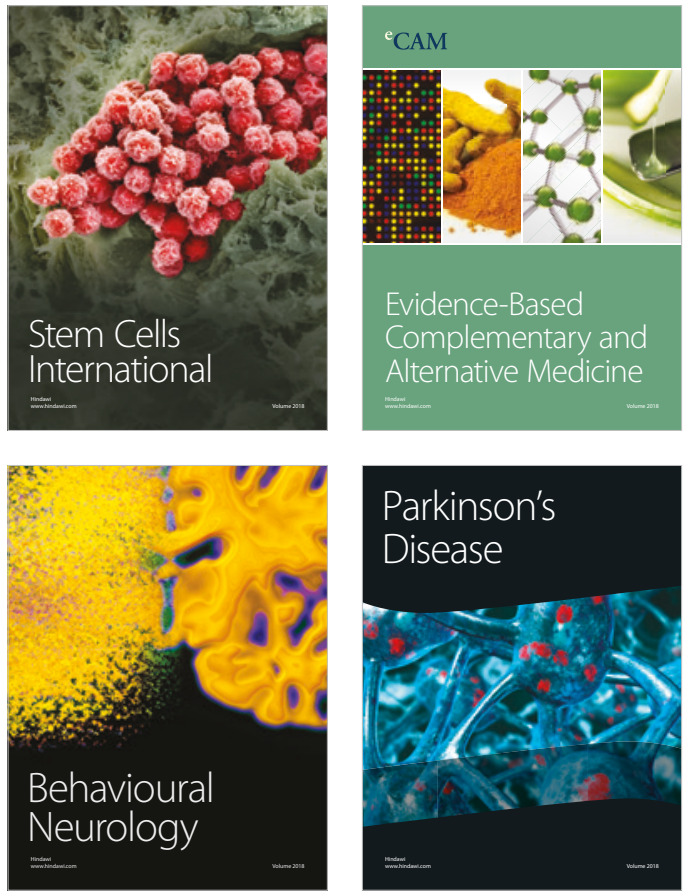

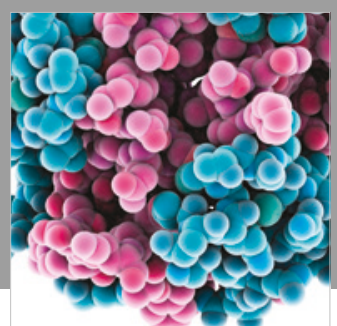

ournal of

Diabetes Research

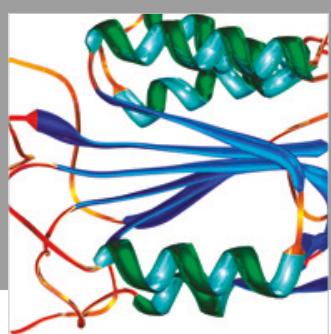

Disease Markers
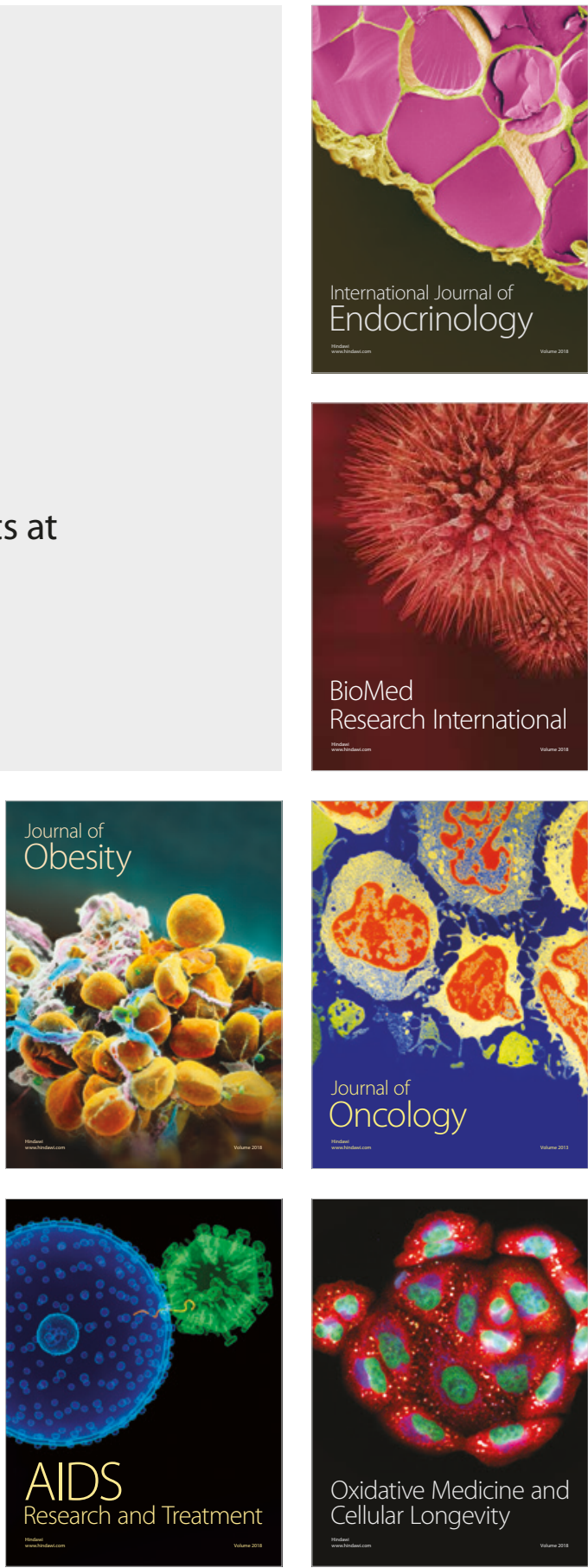